\title{
Research on time synchronization technology of virtual radio
}

\author{
Han Bingyin \\ Department of Optical and Electrical Equipment \\ Academy of Equipment \\ Beijing, China \\ e-mail: hanbingyin@foxmail.com
}

\author{
Zheng HaiXin \\ Department of Optical and Electrical Equipment \\ Academy of Equipment \\ Beijing, China \\ e-mail: zheng.haixin@foxmail.com
}

\author{
Wang Yuanqin \\ Department of Optical and Electrical Equipment \\ Academy of Equipment \\ Beijing, China \\ e-mail: wangyuanqin@yahoo.com
}

\begin{abstract}
The time synchronization in the virtual radio system is determined as one of thorny technical problems. Especially in TT\&C (Telemetry、Tracking and Command) system, it requires incredible precision with time. A novel method for time synchronization by high-precision in a unified virtual radio model specially designed is derived based on AD/DA cache and software cache in the present study. The unified virtual radio model with distributed frame is designed for most applications of virtual radio. And on this basis, the time synchronization strategy is detailed on time synchronization strategy from two designs of daughter card and front-end computer software. Finally, it is also discussed preliminary that time delay of demodulation for this model have an effect on radio system (TT\&C).
\end{abstract}

\author{
Gu Xianglong \\ Department of Optical and Electrical Equipment \\ Academy of Equipment \\ Beijing, China \\ e-mail: guxianglong8345@qq.com
}

Keywords-component; system of signal processing; Aerospace Measurement; Aerospace control; virtual radio; Time synchronization

\section{INTRODUCTION}

Virtual radio is software radio in the advanced stage. It is proposed by Vanu G. Bose et al. at the Massachusetts Institute of Technology [1] in 1999. The basic model is shown in Fig .1.

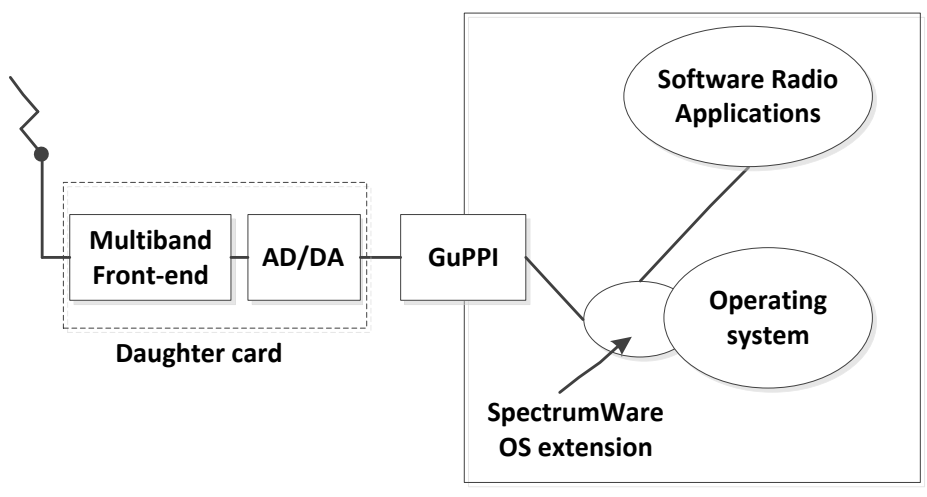

Figure 1. Virtual radio model

Virtual radio contains multiband analog front end and $\mathrm{AD} / \mathrm{DA}$ converter, general interface of $\mathrm{I} / \mathrm{O}$, general computer hardware and software applications. It uses general-purpose computers designed to be more flexible for radio system. Generally, virtual radio has some advantages over traditional software radio, such as rapid configuration, integration with applications, low cost, convenient to upgrade, etc. So, with the advancement of related technology, virtual radio is the future development direction of the software radio.

In traditional software radio platform[2-3], the core of signal processing is performed by DSP and FPGA chip. The chip is driven by high speed clock and processing time is relatively accurate (Accuracy is determined by the clock rate and usually comes to be nanosecond) [4]. In virtual radio platform, the core part for signal processing is composed of shelf type general computers. Under the existing framework, in which task management is 
dependent on the operating system, accuracy of programs' running-time is millisecond. It is not suitable for highspeed signal processing. Therefore, it is essential to study a virtual radio unified model with enough accuracy of time synchronization.

\section{UNIFIED MODEL OF VIRTUAL RADIO}

On the basis of summarizing the predecessors' research [5-6], combined with the current status of related technologies[7-11], following with the basic characteristics of virtual radio [1], considering of the problem of time synchronization in virtual radio system, the basic structure of virtual radio is design as shown in Fig .2.

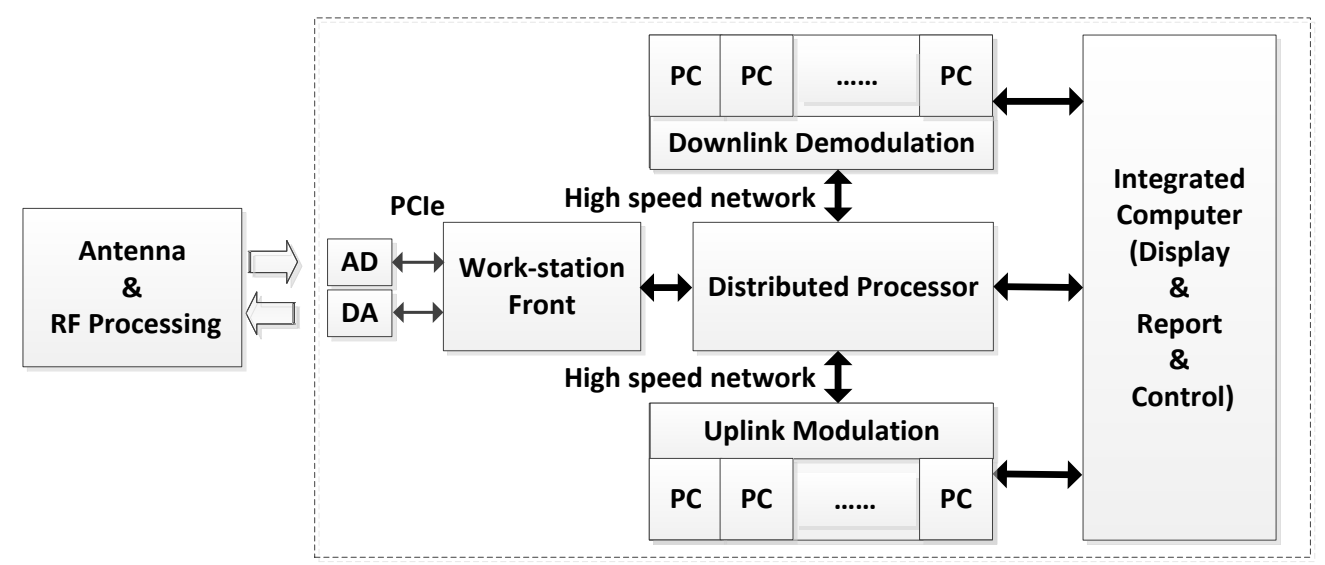

Figure 2. The basic structure of virtual radio

Note that virtual radio platform means the baseband processing parts indicated in the dashed frame on the drawing of Fig .2. The virtual radio platform in this design consist of front-end computer with daughter card, distributing computer, uplink modulation computers, downlink demodulation computers and terminal computes

All the computers send and receive data via a highspeed network. The front-end computer with daughter card realizes conversion between baseband digital signal and analog signal. In distributing computer, the various functions include dataflow management, status monitoring, parameter configuration, information processing and applications are achieved. In uplink modulation computers, uplink baseband signal is generated according to the modulation parameters. Downlink demodulation computers is the major component of signal processing, and all the functions of baseband signal demodulation are achieved in them. Though the terminal computes users can interact with virtual radio platform, such as status display, record the inventory and command control, etc.

\section{TIME SYNCHRONIZATION STRATEGY}

Signal processing in virtual radio system is based on general purpose computer with numerous interface and complex Operating System. Due to computer usually working in multi-task mode managed by Operating System, execution time of one task is uncontrollable. Even (What's more), some task may be delayed by interrupt request of interface. Therefore, it is difficult to ensure the stability rate of both data transmission and signal processing for virtual radio system. Low time accuracy of signal processing execution cannot meet requirements of time synchronization in TT\&C system. The solution in this article is that specialized cache structure of software and hardware is designed to smooth data transmission bandwidth, and specialized manage software for the cache is designed to ensure time synchronization.

\section{A. Daughter card design}

As is different from that mentioned in [12], virtual radio contains a specialized daughter card based on PCIE interface in order to adapt itself to the requirements of time synchronization. The basic principle of daughter card is shown in Fig .3.

\section{B. Software design}

According to the describes of the virtual radio's structure in Section II, the main function of software on front-end computer is to stabilize data transmission and to be ensure of time synchronization through a special kind of data management mechanism. In the scheme of time synchronization, software on front-end computer is driven by AD/DA data. So, initialization software should be start firstly. The initialization process of software is shown in Fig .5. 


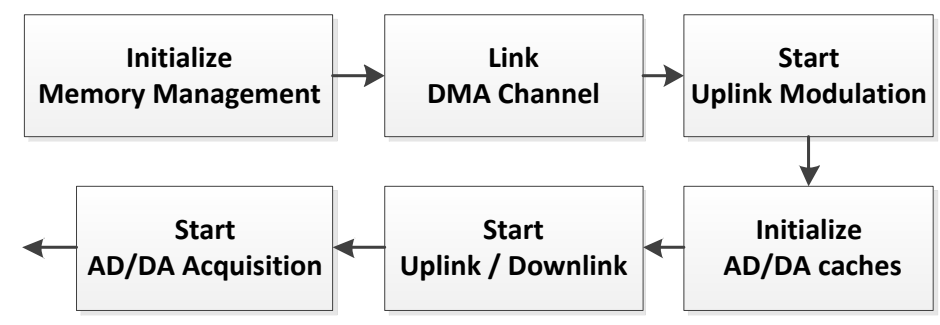

Figure 3. The initialization process of software

When initialization is complete, software will be in a state of multitasking. A cache region ( $\mathrm{N}$ groups of data cache) is established in memory, which is used to cache $\mathrm{AD} / \mathrm{DA}$ data. The data length of each group is consistent with the cache size of each group in daughter card. The structure of data cache is shown in Fig .6. Fig .6 (a) shows data cache in the state of initialization. Fig .6 (b) shows data cache in the state of running. $P_{\mathrm{DA}}$ is a pointer of DA data cache towards DMA transfer. $P_{\text {uplink }}$ is a pointer of DA data cache from uplink data buffer in distribution computer. $P_{\text {downlink }}$ is a pointer of AD data cache towards downlink data buffer in distribution computer. $P_{\mathrm{AD}}$ is a pointer of AD data cache from DMA transfer. Positions of all pointers move in a clockwise direction.

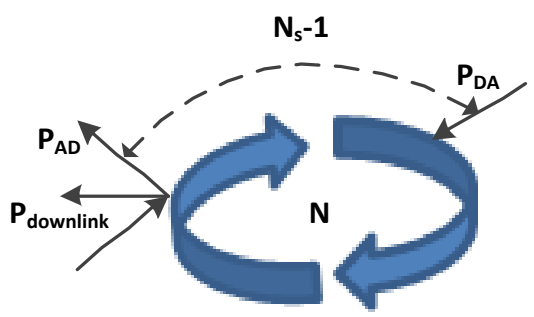

(a)

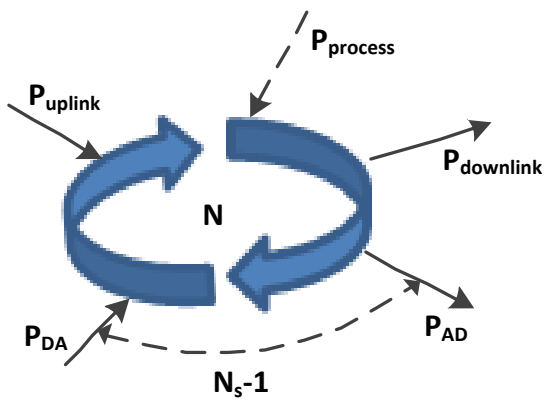

(b)

Figure 4. The structure of data cache

Considering the actual case in TT\&C system, the basic idea of radio ranging is to relate downlink signal with uplink signal for path delay which is used to complete the preliminary ranging.

Software on front-end computer caches $\mathrm{N}$ groups of data, and distributes to the downlink computers and integrated computer. Each group of data contains the cache information such as a number. This is the key point of time synchronization. The frame format of network data is shown in TAB. 1. As it is shown in TAB. 1, sharing parameter contains data length in each group, modulation and demodulation parameters, network data transmission frame format, etc.

TABLE I. THE FRAME FORMAT OF NETWORK DATA

\begin{tabular}{|l|l|l|l|l|l|l|l|}
\hline Format 1 & Cache number & Type of data (uplink) & Data & Initial phase & Blank & Blank \\
\hline Format 2 & Cache number & Type of data (downlink) & Data & Blank & Blank & Blank \\
\hline Format 3 & Cache number & Type of data (uplink) & Blank & Initial phase & Blank & Blank \\
\hline Format 4 & Cache number & Type of data (downlink) & Blank & Initial phase & Doppler & Telemetry frame & Sharing parameter \\
\hline
\end{tabular}

In this paper, design of virtual radio measurement and control system using the network distributed architecture, data transmission between computer need to occupy a certain bus bandwidth and network transmission bandwidth, which requires on the premise of meet the function of measurement and control system, computer data transmission between the transmission bandwidth saving as much as possible. The main network transmission data format is shown in Fig .5. 


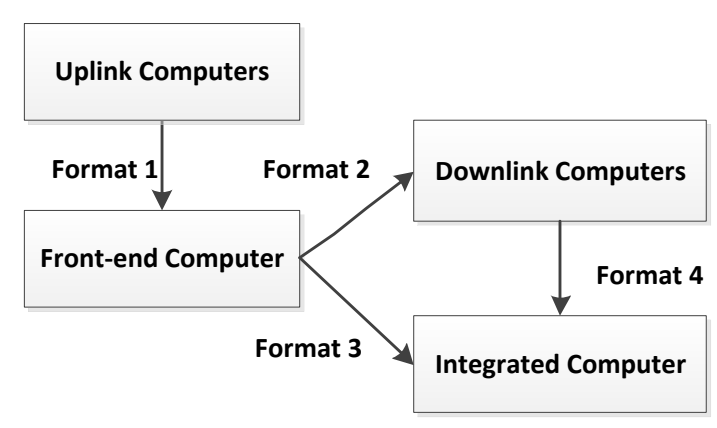

Figure 5. The main network transmission data format

While demodulation is done by downlink computer, integrated can realize ranging with the corresponding cache number related. In order to make the system work stably, software should keep three states be normal.

(A). Keep daughter card and DMA transfer be normal.

According to the working principle and software acquisition card cache structure, both in initial state and running state should satisfy the following condition: $\left(P_{\mathrm{DA}}-P_{\mathrm{AD}}\right)_{N}=N_{s}-1$ (unit is group of cache, $(\mathrm{g})_{N}$ represents remainder of Cycle).

(B). Keep generating uplink signals and network state be normal.

First of all, data written to DA cache is uplink modulation data, and is send from uplink computers to front-end computer. Here is the condition: $P_{\text {uplink }}>P_{\mathrm{DA}}$. Furthermore, in TT\&C system ranging is to make a correlation between uplink data and downlink data. Here is the condition: $P_{\text {process }}>P_{\text {uplink }}$. Above all, it is the condition: $P_{\text {process }}>P_{\text {uplink }}>P_{\mathrm{DA}}$.

(C) Keep generating down signals and network state be normal.

Similarly, it's the condition: $P_{\text {process }}<P_{\text {downlink }}<P_{\mathrm{AD}}$.

\section{PERFORMANCE}

It is not the only way in the paper to realize time synchronization in virtual radio. However, there are several advantages to take this scheme. Firstly, it is a common approach to solve the problem of time synchronization in virtual radio. Secondly, it is able to buffer unstable factors of signal processing though data caches. What's more, the underlying implementation is shielded in this scheme. As every coin has two sides, it is the primary problem associated with it that the system demodulation delay increases.

The system demodulation delay mainly derives from data caches and data demodulation. Therefore, factors of possible delay contain data cache in daughter card, software cache in the front-end computer and downlink data cache for demodulating.

Data in daughter card is transferred to memory in the front-end computer by interrupt. The interval between interrupts, which is supposed as delay of $N_{D}$ groups of data sampling time, is the ideal delay in daughter card. The size of software cache in the front-end computer is
$P_{\text {downlink }}-P_{\mathrm{AD}}$ groups of data. It caches for demodulating. And as we know, the demodulating in software is block by block. It shows that the delay of software cache in the front-end computer is almost the same as the delay of downlink data cache for demodulating. Thus, the ideal system demodulation delay is delay of $N_{D}$ groups of data sampling time and delay of $P_{\text {downlink }}-P_{\mathrm{AD}}$ groups of data sampling time.

Compared with traditional software radio TT\&C, the system demodulation delay increases by amount of data caches. This part of delay is corresponded with the buffer size. And the buffer size and the system stability are complementary. Therefore, it should be perfectly balanced them in practice.

The delay by retaining which it could keep the system stable is defined as the minimum system demodulation delay. It can be an important reference index as evaluation of a virtual radio system.

\section{CONCLUSION}

According to the problem of time synchronization in virtual radio TT\&C, a unified model of virtual radio with time synchronization is proposed in this paper. The distributed structure of virtual radio is adopted. It is an open, extensible in signal processing ability. Though establishing the reasonable structure of hardware and software cache, it has not yet achieved time synchronization, but has stabilized transmission bandwidth. The delay of demodulation in virtual radio and minimum system demodulation delay are also pointed out. It is provided as a reference for related research.

\section{REFERENCES}

[1] Vanu G. Bose. Design and Implementation of Software Radios Using a General Purpose Processor[D]. Massachusetts Institute of Technology, 1999.

[2] WANG Qingsheng. "Design of Software Radio System Based on SCA Specifications", Modern Navigation, vol.01, pp. 56-60, 2012

[3] TAN Zuo-hong;TIAN Zeng-shan et al. "The Design of FPGA + DSP-based High-speed Baseband Signal Processing Platform ", Science Technology and Engineering, vol.03, pp. 239-242, 2014

[4] Joe Mitola. The Software Radio Architecture[J]. IEEE Communications Magzine, 1995, 33(5):26-38.

[5] J. Kumagai, “Winner: Radio Revolutionaries,” IEEE Spectrum, Jan. 2007.

[6] Kan ZHENG, "Open wireless software radio on common pc", The 17th Annual IEEE International Symposium on Personal, Indoor and Mobile Radio Communications (PIMRC' 06). 2006.

[7] Vanu Website[EB/OL]. http://www.vanu.com, 2014

[8] Michael L. Dickens, Brian P. Dunn, and J. Nicholas Laneman. "Design and Implementation of a Portable Software Radio", 2008.

[9] Fang Yi. Research and Development of a Multi-core CPU based Software Radio Platform and Application[D]. Beijing Jiaotong University, 2013

[10] Katabi D. Sora promises lasting impact: technical perspective[J]. Communications of the ACM, 2011, 54(1): 98-98.

[11] Shepard C, Yu H, Anand N, et al. "Argos: practical many-antenna base stations". Proceedings of the 18th annual international conference on Mobile computing and networking, New York, NY USA: ACM, 2012. 53-64.

[12] Microsoft Research Software Radio[EB/OL]. http://research.microsoft.com, 2014 